\title{
NAVIER-STOKES EQUATIONS IN ARBITRARY DOMAINS : THE FUJITA-KATO SCHEME
}

\author{
Sylvie Monniaux
}

\begin{abstract}
Navier-Stokes equations are investigated in a functional setting in 3D open sets $\Omega$, bounded or not, without assuming any regularity of the boundary $\partial \Omega$. The main idea is to find a correct definition of the Stokes operator in a suitable Hilbert space of divergence-free vectors and apply the Fujita-Kato method, a fixed point procedure, to get a local strong solution.
\end{abstract}

\section{Introduction}

Since the pioneering work by Leray [3] in 1934, there have been several studies on solutions of Navier-Stokes equations

$$
(N S)\left\{\begin{array}{rllll}
\frac{\partial u}{\partial t}-\Delta u+\nabla \pi+(u \cdot \nabla) u & =0 & \text { in } & ] 0, T[\times \Omega, \\
\operatorname{div} u & =0 & \text { in }] 0, T[\times \Omega, \\
u & =0 & \text { on }] 0, T[\times \partial \Omega, \\
u(0) & =u_{0} & \text { in } & \Omega .
\end{array}\right.
$$

Fujita and Kato [2] in 1964 gave a method to construct so called mild solutions in smooth domains $\Omega$, producing local (in time) smooth solutions of $(N S)$ in a Hilbert space setting. These solutions are global in time if the initial value $u_{0}$ is small enough in a certain sense. The case of non smooth domains has been studied by Deuring and von Wahl [1] in 1995 where they considered domains $\Omega \subset \mathbb{R}^{3}$ with Lipschitz boundary $\partial \Omega$. They found local smooth solutions using results contained in Shen's $\mathrm{PhD}$ thesis [4]. Their method does not cover the critical space case as in [2]. One of the difficulty there was to understand the Stokes operator, and in particular its domain of definition.

In Section 2, we give a "universal" definition of the Stokes operator, for any domain $\Omega \subset \mathbb{R}^{3}$ (Defintion 2.4). In Section 3, we construct a mild solution of $(N S)$ with a method similar to Fujita-Kato's [2] (Theorem 3.5) for initial values $u_{0}$ in the critical space $D\left(A^{\frac{1}{4}}\right)$. We show in Section 4 that this mild solution is a strong solution, i.e. $(N S)$ is satisfied almost everywhere.

\section{The Stokes operator}

Let $\Omega$ be an open set in $\mathbb{R}^{3}$. The space

$$
L^{2}(\Omega)^{3}=\left\{u=\left(u_{1}, u_{2}, u_{3}\right) ; u_{i} \in L^{2}(\Omega), i=1,2,3\right\}
$$

Received by the editors November 15, 2005.

2000 Mathematics Subject Classification. Primary 35Q10, 76D05 ; Secondary 35A15. 
endowed with the scalar product

$$
\langle u, v\rangle=\int_{\Omega} u \cdot \bar{v}=\sum_{i=1}^{3} \int_{\Omega} u_{i} \overline{v_{i}}
$$

is a Hilbert space. Define

$$
\mathcal{G}=\left\{\nabla p ; p \in L_{l o c}^{2}(\Omega) \text { with } \nabla p \in L^{2}(\Omega)^{3}\right\} ;
$$

the set $\mathcal{G}$ is a closed subspace of $L^{2}(\Omega)^{3}$. Let

$$
\mathcal{H}=\mathcal{G}^{\perp}=\left\{u \in L^{2}(\Omega)^{3} ;\langle u, \nabla p\rangle=0, \forall p \in L_{l o c}^{2}(\Omega) \text { with } \nabla p \in L^{2}(\Omega)^{3}\right\} .
$$

The space $\mathcal{H}$, endowed with the scalar product $\langle\cdot, \cdot\rangle$ is a Hilbert space. We have the following Hodge decomposition

$$
L^{2}(\Omega)^{3}=\mathcal{H} \stackrel{\perp}{\oplus} \mathcal{G} .
$$

We denote by $\mathbb{P}$ the projection from $L^{2}(\Omega)^{3}$ onto $\mathcal{H}: \mathbb{P}$ is the usual Helmoltz projection. We denote by $J$ the canonical injection $\mathcal{H} \hookrightarrow L^{2}(\Omega)^{3}: J^{\prime}=\mathbb{P}\left(J^{\prime}\right.$ beeing the adjoint of $J$ ) and $\mathbb{P} J$ is the identity on $\mathcal{H}$. Let now $\mathscr{D}(\Omega)^{3}=\mathscr{C}_{c}^{\infty}(\Omega)^{3}$ and

$$
\mathcal{D}=\left\{u \in \mathscr{D}(\Omega)^{3} ; \operatorname{div} u=0\right\} .
$$

It is clear that $\mathcal{D}$ is a closed subspace of $\mathscr{D}(\Omega)^{3}$. We denote by $J_{0}: \mathcal{D} \hookrightarrow \mathscr{D}(\Omega)^{3}$ the canonical injection : $J_{0} \subset J$. Let $\mathbb{P}_{1}$ be the adjoint of $J_{0}: \mathbb{P}_{1}=J_{0}^{\prime}: \mathscr{D}^{\prime}(\Omega)^{3} \rightarrow \mathcal{D}^{\prime}$. We have $\mathbb{P} \subset \mathbb{P}_{1}$. The following theorem characterizes the elements in ker $\mathbb{P}_{1}$.

Theorem 2.1 (de Rham). Let $T \in \mathscr{D}^{\prime}(\Omega)^{3}$ such that $\mathbb{P}_{1} T=0$ in $\mathcal{D}^{\prime}$. Then there exists $S \in\left(\mathscr{C}_{c}^{\infty}(\Omega)\right)^{\prime}$ such that $T=\nabla S$. Conversely, if $T=\nabla S$ with $S \in\left(\mathscr{C}_{c}^{\infty}(\Omega)\right)^{\prime}$, then $\mathbb{P}_{1} T=0$ in $\mathcal{D}^{\prime}$.

We denote by $H_{0}^{1}(\Omega)^{3}$ the closure of $\mathscr{D}(\Omega)^{3}$ with respect to the scalar product $(u, v) \mapsto\langle u, v\rangle_{1}=\langle u, v\rangle+\sum_{i=1}^{3}\left\langle\partial_{i} u, \partial_{i} v\right\rangle$. By Sobolev embeddings, we have $H_{0}^{1}(\Omega)^{3} \hookrightarrow L^{6}(\Omega)^{3}$. Define

$$
\mathcal{V}=\mathcal{H} \cap H_{0}^{1}(\Omega)^{3} .
$$

The space $\mathcal{V}$ is a closed subspace of $H_{0}^{1}(\Omega)^{3}$; endowed with the scalar product $\langle\cdot, \cdot\rangle_{1}$, $\mathcal{V}$ is a Hilbert space.

Proposition 2.2. The space $\mathcal{V}$ is dense in $\mathcal{H}$.

Proof. Let $u \in \mathcal{H}$ be in the orthogonal of $\mathcal{V}$ with respect to $\mathcal{H}$, i.e.

$$
\langle u, v\rangle=0 \quad \text { for all } v \in \mathcal{V} .
$$

Since $\mathcal{D} \subset \mathcal{V},(2.1)$ implies also

$$
\langle u, v\rangle=0 \quad \text { for all } v \in \mathcal{D} .
$$

It means that $u$, viewed as an element of $\mathcal{D}^{\prime}$, is 0 . By Theorem 2.1, there exists a distribution $S \in \mathscr{D}(\Omega)^{\prime}$ such that $J u=\nabla S$. Since $J u \in L^{2}(\Omega)^{3}$, so is $\nabla S$ and therefore, $u=\mathbb{P} J u=\mathbb{P} \nabla S=0$. 
The canonical injection $\tilde{J}: \mathcal{V} \hookrightarrow H_{0}^{1}(\Omega)^{3}$ is the restriction of $J$ to $\mathcal{V}$. We denote by $\tilde{\mathbb{P}}$ the adjoint of $\tilde{J}$ : since $\tilde{J}$ is the restriction of $J$ to $\mathcal{V}, \tilde{\mathbb{P}}$ is an extension of $\mathbb{P}$ to $\mathcal{V}^{\prime}$. On $\mathcal{V} \times \mathcal{V}$ we define now the form $a$ by $a(u, v)=\sum_{i=1}^{3}\left\langle\partial_{i} \tilde{J} u, \partial_{i} \tilde{J} v\right\rangle: a$ is a bilinear, symmetric, $\delta+a$ is a coercive form on $\mathcal{V} \times \mathcal{V}$ for all $\delta>0$, then defines a bounded self-adjoint operator $A_{0}: \mathcal{V} \rightarrow \mathcal{V}^{\prime}$ by $\left(A_{0} u\right)(v)=a(u, v)$ with $\delta+A_{0}$ invertible for all $\delta>0$.

Proposition 2.3. For all $u \in \mathcal{V}, A_{0} u=\tilde{\mathbb{P}}\left(-\Delta_{D}^{\Omega}\right) \tilde{J} u$, where $\Delta_{D}^{\Omega}$ denotes the DirichletLaplacian on $H_{0}^{1}(\Omega)^{3}$.

Proof. For all $u, v \in \mathcal{V}$, we have

$$
\begin{aligned}
\left(A_{0} u\right)(v) & \stackrel{(1)}{=} a(u, v) \stackrel{(2)}{=} \sum_{i=1}^{3}\left\langle\partial_{i} \tilde{J} u, \partial_{i} \tilde{J} v\right\rangle \\
& \stackrel{(3)}{=}\left\langle\left(-\Delta_{D}^{\Omega}\right) \tilde{J} u, \tilde{J} v\right\rangle_{H^{-1}, H_{0}^{1}} \\
& \stackrel{(4)}{=} \quad\left\langle\tilde{\mathbb{P}}\left(-\Delta_{D}^{\Omega}\right) \tilde{J} u, v\right\rangle_{\mathcal{V}^{\prime}, \mathcal{V}}
\end{aligned}
$$

The first two equalities come from the definition of $A_{0}$ and $a$. The third equality comes from the definition of the Dirichlet-Laplacian on $H_{0}^{1}(\Omega)^{3}$ and the fact that for $v \in \mathcal{V}, \tilde{J} v=v$. The last equality is due to $\tilde{J}^{\prime} \varphi=\tilde{\mathbb{P}} \varphi$ in $\mathcal{V}^{\prime}$ for all $\varphi \in H^{-1}(\Omega)^{3}$. This shows that $A_{0} u$ and $\tilde{\mathbb{P}}\left(-\Delta_{D}^{\Omega}\right) \tilde{J} u$ are two continuous linear forms on $\mathcal{V}$ which coïncide on $\mathcal{V}$, they are then equal.

Definition 2.4. The operator $A$ defined on its domain $D(A)=\left\{u \in \mathcal{V} ; A_{0} u \in \mathcal{H}\right\}$ by $A u=A_{0} u$ is called the Stokes operator.

Theorem 2.5. The Stokes operator is self-adjoint in $\mathcal{H}$, generates an analytic semigroup $\left(e^{-t A}\right)_{t \geq 0}, D\left(A^{\frac{1}{2}}\right)=\mathcal{V}$ and satisfies

$$
\begin{aligned}
D(A) & =\left\{u \in \mathcal{V} ; \exists \pi \in\left(\mathscr{C}_{c}^{\infty}(\Omega)\right)^{\prime}: \nabla \pi \in H^{-1}(\Omega) \text { and }-\Delta u+\nabla \pi \in \mathcal{H}\right\} \\
A u & =-\Delta u+\nabla \pi .
\end{aligned}
$$

Remark 2.6. Since $H_{0}^{1}(\Omega)^{3} \hookrightarrow L^{6}(\Omega)^{3}$, it is clear by interpolation and dualization that $\tilde{\mathbb{P}}$ maps $L^{p}(\Omega)^{3}$ to $D\left(A^{s}\right)^{\prime}$ for $\frac{6}{5} \leq p \leq 2,0 \leq s \leq \frac{1}{2}$ and $s=-\frac{3}{4}+\frac{3}{2 p}$. Since $A$ is self-adjoint, one has $\left(\delta+A_{0}\right)^{-s} D\left(A^{s}\right)^{\prime}=\left\{\left(\delta+A_{0}\right)^{-s} u ; u \in D\left(A^{s}\right)^{\prime}\right\}=\mathcal{H}$. In particular, $\left(\delta+A_{0}\right)^{-\frac{1}{4}} \mathbb{P}_{1}$ maps $L^{\frac{3}{2}}(\Omega)^{3}$ into $\mathcal{H}$.

\section{Mild solution to the Navier-Stokes system}

Let $T>0$.

Define the following Banach space

$$
\begin{aligned}
\mathcal{E}_{T}= & \left.\left\{u \in \mathscr{C}\left([0, T] ; D\left(A^{\frac{1}{4}}\right)\right) \cap \mathscr{C}^{1}(] 0, T\right] ; D\left(A^{\frac{1}{4}}\right)\right) \\
& \text { such that } \left.\sup _{0<s<T}\left\|s^{\frac{1}{4}} A^{\frac{1}{2}} u(s)\right\|_{\mathcal{H}}+\sup _{0<s<T}\left\|s A^{\frac{1}{4}} u^{\prime}(s)\right\|_{\mathcal{H}}<\infty\right\}
\end{aligned}
$$


endowed with the norm

$$
\|u\|_{\mathcal{E}_{T}}=\sup _{0<s<T}\left\|A^{\frac{1}{4}} u(s)\right\|_{\mathcal{H}}+\sup _{0<s<T}\left\|s^{\frac{1}{4}} A^{\frac{1}{2}} u(s)\right\|_{\mathcal{H}}+\sup _{0<s<T}\left\|s A^{\frac{1}{4}} u^{\prime}(s)\right\|_{\mathcal{H}} .
$$

Let $\alpha$ be defined by $\alpha(t)=e^{-t A} u_{0}$ where $u_{0} \in D\left(A^{\frac{1}{4}}\right)$. Then $\alpha \in \mathcal{E}_{T}$. Indeed, it is clear that $\alpha \in \mathscr{C}\left([0, T] ; D\left(A^{\frac{1}{4}}\right)\right)$. We also have that $t^{\frac{1}{4}} A^{\frac{1}{2}} \alpha(t)=t^{\frac{1}{4}} A^{\frac{1}{4}} e^{-t A} A^{\frac{1}{4}} u_{0}$ is bounded on $(0, T)$ since $\left(e^{-t A}\right)_{t \geq 0}$ is an analytic semigroup. Moreover, one has $\alpha^{\prime}(t)=-A e^{-t A} u_{0}$ which yields to $t A^{\frac{1}{4}} \alpha^{\prime}(t)=-t A e^{-t A} A^{\frac{1}{4}} u_{0}$ continuous on $\left.] 0, T\right]$, bounded in $\mathcal{H}$. For $u, v \in \mathcal{E}_{T}$, we define now

$$
\Phi(u, v)(t)=\int_{0}^{t} e^{-(t-s) A}\left(-\frac{1}{2} \tilde{\mathbb{P}}\right)((u(s) \cdot \nabla) v(s)+(v(s) \cdot \nabla) u(s)) d s, \quad 0<t<T .
$$

Notation 3.1. Let $X, Y$ be Banach spaces. For a bounded linear operator $S: X \rightarrow$ $Y$, we denote by $\|S\|_{\mathscr{L}(X ; Y)}$ the norm of $S$, i.e.

$$
\|S\|_{\mathscr{L}(X ; Y)}=\sup \left\{\|S x\|_{Y} ; \forall x \in X \text { with }\|x\|_{X} \leq 1\right\} .
$$

If $X=Y$, we adopt the notation $\|S\|_{\mathscr{L}(X)}$ instead of $\|S\|_{\mathscr{L}(X ; Y)}$. For a bilinear operator $B: X \times X \rightarrow Y$, we denote by $\|B\|_{\mathscr{L}(X \times X ; Y)}$ the norm of $B$, i.e.

$\|B\|_{\mathscr{L}(X \times X ; Y)}=\sup \left\{\left\|B\left(x, x^{\prime}\right)\right\|_{Y} ; \forall x, x^{\prime} \in X\right.$ with $\|x\|_{X} \leq 1$ and $\left.\left\|x^{\prime}\right\|_{X} \leq 1\right\}$.

Notation 3.2. For $u, v \in L^{2}(\Omega)^{3}$, we denote by $u \otimes v$ the matrix defined by

$$
(u \otimes v)_{i, j}=u_{i} v_{j}, \quad 1 \leq i, j \leq 3 .
$$

Remark 3.3. If $u, v$ are sufficiently smooth vector fields such that $\operatorname{div} u=0$, then

$$
\operatorname{div}(u \otimes v):=\sum_{i=1}^{3} \partial_{i}\left(u_{i} v\right)=\sum_{i=1}^{3} u_{i} \partial_{i} v=(u \cdot \nabla) v
$$

Proposition 3.4. The transform $\Phi$ is bilinear, symmetric, continuous from $\mathcal{E}_{T} \times \mathcal{E}_{T}$ to $\mathcal{E}_{T}$ and the norm of $\Phi$ is independent of $T$.

Proof. The fact that $\Phi$ is bilinear and symmetric is clear. Moreover, $\Phi(u, v)=e^{-\cdot A} * f$, where $f$ is defined by

$$
f(s)=\left(-\frac{1}{2} \tilde{\mathbb{P}}\right)((u(s) \cdot \nabla) v(s)+(v(s) \cdot \nabla) u(s)), \quad s \in[0, T] .
$$

For $u, v \in \mathcal{E}_{T}$, it is clear that $(u(s) \cdot \nabla) v(s)+(v(s) \cdot \nabla) u(s) \in L^{\frac{3}{2}}(\Omega)^{3}$ and therefore $\left(\delta+A_{0}\right)^{-\frac{1}{4}} f(s) \in \mathcal{H}$ with $\sup _{0<s<T} s^{\frac{1}{2}}\left\|\left(\delta+A_{0}\right)^{-\frac{1}{4}} f(s)\right\|_{\mathcal{H}} \leq c\|u\|_{\mathcal{E}_{T}}\|v\|_{\mathcal{E}_{T}}$. We have then

$$
\Phi(u, v)=e^{-\cdot A} * f=(\delta+A)^{\frac{1}{4}} e^{-\cdot A} *\left(\left(\delta+A_{0}\right)^{-\frac{1}{4}} f\right)
$$

and therefore

$$
\begin{aligned}
\left\|A^{\frac{1}{4}} \Phi(u, v)(t)\right\|_{\mathcal{H}} & \leq \int_{0}^{t}\left\|A^{\frac{1}{4}}(\delta+A)^{\frac{1}{4}} e^{-(t-s) A}\right\|_{\mathscr{L}(\mathcal{H})}\left\|\left(\delta+A_{0}\right)^{-\frac{1}{4}} f(s)\right\|_{\mathcal{H}} d s \\
& \leq c\left(\int_{0}^{t} \frac{1}{\sqrt{t-s}} \frac{1}{\sqrt{s}} d s\right)\|u\|_{\mathcal{E}_{T}}\|v\|_{\mathcal{E}_{T}} \\
& \leq c\left(\int_{0}^{1} \frac{1}{\sqrt{1-\sigma}} \frac{1}{\sqrt{\sigma}} d \sigma\right)\|u\|_{\mathcal{E}_{T}}\|v\|_{\mathcal{E}_{T}} \\
& \leq c\|u\|_{\mathcal{E}_{T}}\|v\|_{\mathcal{E}_{T}} .
\end{aligned}
$$


Continuity with respect to $t \in[0, T]$ of $t \mapsto A^{\frac{1}{4}} \Phi(u, v)(t)$ is clear once we have proved the boundedness. We also have

$$
\begin{aligned}
\left\|A^{\frac{1}{2}} \Phi(u, v)(t)\right\|_{\mathcal{H}} & \leq \int_{0}^{t}\left\|A^{\frac{1}{2}}(\delta+A)^{\frac{1}{4}} e^{-(t-s) A}\right\|_{\mathscr{L}(\mathcal{H})}\left\|\left(\delta+A_{0}\right)^{-\frac{1}{4}} f(s)\right\|_{\mathcal{H}} d s \\
& \leq c\left(\int_{0}^{t} \frac{1}{(t-s)^{\frac{3}{4}}} \frac{1}{\sqrt{s}} d s\right)\|u\|_{\mathcal{E}_{T}}\|v\|_{\mathcal{E}_{T}} \\
& \leq c t^{-\frac{1}{4}}\left(\int_{0}^{1} \frac{1}{(1-\sigma)^{\frac{3}{4}}} \frac{1}{\sqrt{\sigma}} d \sigma\right)\|u\|_{\mathcal{E}_{T}}\|v\|_{\mathcal{E}_{T}} \\
& \leq c t^{-\frac{1}{4}}\|u\|_{\mathcal{E}_{T}}\|v\|_{\mathcal{E}_{T}} .
\end{aligned}
$$

Continuity with respect to $t \in] 0, T]$ is clear once we have proved the boundedness. To prove the last part of the norm of $\Phi(u, v)$ in $\mathcal{E}_{T}$, we first write $f$, using Notation 3.2 and Remark 3.3, in the following form

$$
f(s)=\left(-\frac{1}{2} \tilde{\mathbb{P}}\right) \operatorname{div}(u(s) \otimes v(s)+v(s) \otimes u(s)), \quad s \in[0, T] .
$$

We have then for $s \in] 0, T[$

$$
f^{\prime}(s)=\left(-\frac{1}{2} \tilde{\mathbb{P}}\right) \operatorname{div}\left(u^{\prime}(s) \otimes v(s)+u(s) \otimes v^{\prime}(s)+v^{\prime}(s) \otimes u(s)+v(s) \otimes u^{\prime}(s)\right) .
$$

For all $s \in] 0, T]$ we have

$$
\begin{aligned}
s^{\frac{5}{4}}\left\|u^{\prime}(s) \otimes v(s)\right\|_{2} & \stackrel{(1)}{\leq}\left\|s u^{\prime}(s)\right\|_{3}\left\|s^{\frac{1}{4}} v(s)\right\|_{6} \\
& \stackrel{(2)}{\leq}\left\|s A^{\frac{1}{4}} u^{\prime}(s)\right\|_{\mathcal{H}}\left\|s^{\frac{1}{4}} A^{\frac{1}{2}} v(s)\right\|_{\mathcal{H}} \\
& \stackrel{(3)}{\leq}\|u\|_{\mathcal{E}_{T}}\|v\|_{\mathcal{E}_{T}},
\end{aligned}
$$

where the first inequality comes from the fact that $L^{3} \cdot L^{6} \hookrightarrow L^{2}$, the second comes from the inclusions $D\left(A^{\frac{1}{4}}\right) \hookrightarrow L^{3}(\Omega)^{3}$ and $D\left(A^{\frac{1}{2}}\right) \hookrightarrow L^{6}(\Omega)^{3}$ and the third inequality follows directly from the definition of the space $\mathcal{E}_{T}$. Of course the same occurs for the other three terms $u(s) \otimes v^{\prime}(s), v^{\prime}(s) \otimes u(s)$ and $v(s) \otimes u^{\prime}(s)$. Therefore, since $A_{0}^{-\frac{1}{2}}$ maps $\mathcal{V}^{\prime}$ to $\mathcal{H}$, we obtain

$$
\sup _{0<s<T}\left\|s^{\frac{5}{4}}\left(\delta+A_{0}\right)^{-\frac{1}{2}} f^{\prime}(s)\right\|_{\mathcal{H}} \leq c\|u\|_{\mathcal{E}_{T}}\|v\|_{\mathcal{E}_{T}} .
$$

We have

$$
\left.\Phi(u, v)(t)=\int_{0}^{\frac{t}{2}} e^{-s A} f(t-s) d s+\int_{0}^{\frac{t}{2}} e^{-(t-s) A} f(s) d s \quad t \in\right] 0, T[,
$$

and therefore

$$
\begin{aligned}
\Phi(u, v)^{\prime}(t)= & e^{-\frac{t}{2} A} f\left(\frac{t}{2}\right)+\int_{0}^{\frac{t}{2}}(\delta+A)^{\frac{1}{2}} e^{-s A}\left(\delta+A_{0}\right)^{-\frac{1}{2}} f^{\prime}(t-s) d s \\
& +\int_{0}^{\frac{t}{2}}-A(\delta+A)^{\frac{1}{4}} e^{-(t-s) A}\left(\delta+A_{0}\right)^{-\frac{1}{4}} f(s) d s,
\end{aligned}
$$


which yields

$$
\begin{aligned}
\left\|A^{\frac{1}{4}} \Phi(u, v)^{\prime}(t)\right\|_{\mathcal{H}} \leq & \frac{c}{\sqrt{t}}\left\|\left(\delta+A_{0}\right)^{-\frac{1}{4}} f\left(\frac{t}{2}\right)\right\|_{\mathcal{H}}+c\left(\int_{0}^{\frac{t}{2}} \frac{1}{s^{\frac{1}{2}}} \frac{1}{(t-s)^{\frac{5}{4}}} d s\right)\|u\|_{\mathcal{E}_{T}}\|v\|_{\mathcal{E}_{T}} \\
& +c\left(\int_{0}^{\frac{t}{2}} \frac{1}{(t-s)^{\frac{5}{4}}} \frac{1}{s^{\frac{1}{2}}} d s\right)\|u\|_{\mathcal{E}_{T}}\|v\|_{\mathcal{E}_{T}} \\
\leq & \frac{c}{t}\left(\int_{0}^{\frac{1}{2}} \frac{d \sigma}{(1-\sigma)^{\frac{5}{4}} \sigma^{\frac{1}{2}}}\right)\|u\|_{\mathcal{E}_{T}}\|v\|_{\mathcal{E}_{T}} .
\end{aligned}
$$

This last inequality ensures that $\Phi(u, v) \in \mathcal{E}_{T}$ whenever $u, v \in \mathcal{E}_{T}$.

Theorem 3.5. For all $u_{0} \in D\left(A^{\frac{1}{4}}\right)$, there exists $T>0$ such that there exists a unique $u \in \mathcal{E}_{T}$ solution of $u=\alpha+\Phi(u, u)$ on $[0, T]$. This function $u$ is called the mild solution to the Navier-Stokes system.

Proof. Let $T>0$. Since $\Phi: \mathcal{E}_{T} \times \mathcal{E}_{T} \rightarrow \mathcal{E}_{T}$ is bilinear continuous, it suffices to apply Picard fixed point theorem, as in [2]. The sequence in $\mathcal{E}_{T}\left(v_{n}\right)_{n \in \mathbb{N}}$ defined by $v_{0}=\alpha$ as first term and

$$
v_{n+1}=\alpha+\Phi\left(v_{n}, v_{n}\right), \quad n \in \mathbb{N}
$$

converges to the unique solution $u \in \mathcal{E}_{T}$ of $u=\alpha+\Phi(u, u)$ provided $\left\|A^{\frac{1}{4}} u_{0}\right\|_{\mathcal{H}}$ is small enough $\left(\|\alpha\|_{\mathcal{E}_{T}}<\frac{1}{4\|\Phi\|_{\mathscr{L}\left(\mathcal{E}_{T} \times \mathcal{E}_{T} ; \mathcal{E}_{T}\right)}}\right)$. In the case where $\left\|A^{\frac{1}{4}} u_{0}\right\|_{\mathcal{H}}$ is not small (that is, if $\|\alpha\|_{\mathcal{E}_{T}} \geq \frac{1}{4\|\Phi\| \mathscr{L}\left(\mathcal{E}_{T} \times \mathcal{E}_{T} ; \mathcal{E}_{T}\right)}$ ) then for $\varepsilon>0$, there exists $u_{0, \varepsilon} \in D(A)$ such that $\left\|A^{\frac{1}{4}}\left(u_{0}-u_{0, \varepsilon}\right)\right\|_{\mathcal{H}} \leq \varepsilon$. If we take as initial value $u_{0, \varepsilon} \in D(A)$, we have

$$
\left\|\alpha_{\varepsilon}\right\|_{\mathcal{E}_{T}} \leq c T^{\frac{3}{4}}\left\|A u_{0, \varepsilon}\right\|_{\mathcal{H}} \underset{T \rightarrow 0}{\longrightarrow} 0 .
$$

Therefore, we can find $T>0$ such that $\|\alpha\|_{\mathcal{E}_{T}}<\frac{1}{4\|\Phi\| \mathscr{L}_{\left(\mathcal{E}_{T} \times \mathcal{E}_{T} ; \mathcal{E}_{T}\right)}}$.

\section{Strong solutions}

Let $u$ be the mild solution to the Navier-Stokes system. We show in this section that $u$ in fact satisfies the equations of the Navier-Stokes system in an $L^{p}$-sense (for a suitable $p$ ). To begin with, we know that $u \in \mathcal{E}_{T}$ and satisfies

$$
u=\alpha+\Phi(u, u)=\alpha+e^{-\cdot A} * \varphi(u),
$$

where $\varphi(u)=-\tilde{\mathbb{P}}((u \cdot \nabla) u)$ and we have $\left\|t^{\frac{1}{2}}(u(t) \cdot \nabla) u(t)\right\|_{\frac{3}{2}} \leq c\|u\|_{\mathcal{E}_{T}}^{2}$. Therefore, we get

$$
\begin{gathered}
u(0)=\alpha(0)=u_{0}, \\
\left.\operatorname{div} u(t)=0 \text { in the } L^{2}-\text { sense for } t \in\right] 0, T[
\end{gathered}
$$

and

$$
u^{\prime}+A u=f \quad \text { in } \mathscr{C}(] 0, T\left[; \mathcal{V}^{\prime}\right)
$$

which means that for all $t \in] 0, T[$,

$$
\tilde{\mathbb{P}}\left(u^{\prime}(t)-\Delta_{D}^{\Omega} u(t)+(u(t) \cdot \nabla) u(t)\right)=0 .
$$


Then, by Theorem 2.1, there exists $(-\pi)(t) \in\left(\mathscr{C}_{c}^{\infty}(\Omega)\right)^{\prime}$ such that $\nabla \pi(t) \in H^{-1}(\Omega)^{3}$ and

$$
\nabla(-\pi)(t)=u^{\prime}(t)-\Delta_{D}^{\Omega} u(t)+(u(t) \cdot \nabla) u(t)
$$

and we have for $0<t<T$

$$
-\Delta_{D}^{\Omega} u(t)+\nabla \pi(t)=-u^{\prime}(t)-(u(t) \cdot \nabla) u(t) \in L^{3}(\Omega)^{3}+L^{\frac{3}{2}}(\Omega)^{3} .
$$

The equation (4.3), together with (4.1) and (4.2), give the usual Navier-Stokes equations which are fulfilled in a strong sense (a.e.) where we consider the expression $-\Delta u+\nabla \pi$ undecoupled.

\section{References}

[1] P. Deuring, W. von Wahl. Strong solutions of the Navier-Stokes system in Lipschitz bounded domains. Math. Nachr. 171 (1995) 111-148.

[2] H. Fujita, T. Kato. On the Navier-Stokes initial value problem I. Arch. Rational Mech. Anal. 16 (194) 269-315.

[3] J. Leray. Sur le mouvement d'un liquide visqueux emplissant l'espace. Acta Math. J. 63 (1934) 193-248.

[4] Z. Shen. Boundary value problems for parabolic Lamé systems and a nonstationary linearized system of Navier-Stokes equations in Lipschitz cylinders. Amer. J. Math. 113 (1991) 293-373.

LATP UMr 6632, Case cour A, Faculté des sciences de Saint-JÉrôme, Université Paul CÉzanne (Aix-Marseille 3), 13397 Marseille Cédex 20, France

E-mail address: sylvie.monniaux@univ.u-3mrs.fr 